\title{
AN EXTREMAL PROBLEM IN UNIFORM DISTRIBUTION THEORY
}

\author{
Vladimír Baláž — Maria Rita Iacò - Oto Strauch - \\ - Stefan Thonhauser - Robert F. Tichy \\ Dedicated to the memory of Professor Pierre Liardet
}

\begin{abstract}
In this paper we consider an optimization problem for Cesàro means of bivariate functions. We apply methods from uniform distribution theory, calculus of variations and ideas from the theory of optimal transport.
\end{abstract}

\section{Communicated by Georg Werner Nowak}

\section{Introduction}

The systematic study of distribution functions of real valued sequences goes back to van der Corput [26, 27. Over the last years these results where extened to multivariate settings by Strauch and several co-authors; see [1, 2, 8, 23. These investigations include the study of the set of all distribution functions of a given sequence and various optimization problems.

A particularly interesting problem is the study of extremal limits of two-dimensional sequences of the form

$$
\frac{1}{N} \sum_{n=1}^{N} F\left(x_{n}, y_{n}\right), \quad N=1,2, \ldots
$$

2010 Mathematics Subject Classification: 11K06, 60E05, 60A10, 49K27.

Keywords: Uniform distribution, copula, Monge-Kantorovich problem, dual problem, $c$-convex function, $c$-subdifferential.

The authors were supported by the bilateral Austria-Slovakia travel grant "Uniform distribution, copulas and applications". The second, forth and fifth authors are supported by the Austrian Science Fund (FWF) Project F5510 (part of the Special Research Program (SFB) "Quasi-Monte Carlo Methods: Theory and Applications"). The second author is also partially supported by the Austrian Science Fund (FWF): W1230, Doctoral Program "Discrete Mathematics". The first and third authors are supported by VEGA Project 2/0149/14. 
where $\left(x_{n}\right)_{n \in \mathbb{N}},\left(y_{n}\right)_{n \in \mathbb{N}}$ are uniformly distributed (for short u.d.) sequences in the unit interval and $F$ is a given continuous function on $[0,1]^{2}$; see $[15$.

Let us recall that a sequence $\left(x_{n}\right)_{n \in \mathbb{N}}$ of points in $[0,1[$ is said to be u.d. if and only if

$$
\lim _{N \rightarrow \infty} \frac{1}{N} \sum_{n=1}^{N} \mathbf{1}_{[a, b[}\left(x_{n}\right)=b-a
$$

for all intervals $\left[a, b\left[\subseteq\left[0,1\left[\right.\right.\right.\right.$, where $\mathbf{1}_{E}$ denotes as usual the indicator function of the set $E$. We refer to [5, 12, 22, as general references on the subject.

A mapping $T$ of the unit interval into itself is called uniform distribution preserving (for short u.d.p.) if the sequence $\left(T\left(x_{n}\right)\right)_{n \in \mathbb{N}}$ is u.d. whenever $\left(x_{n}\right)_{n \in \mathbb{N}}$ is a u.d. sequence in $[0,1[$.

These maps have been extensively studied (see e.g., [4, 16]), also in connection to variational problems [21] and extended to compact metric spaces [24]. They are particularly interesting for the purposes of this paper since they can be thought of as suitable measure preserving rearrangements of the unit interval, as we will see in the next section.

It turned out that the study of the asymptotic behaviour of mean values (1) is equivalent to find optimal bounds for Riemann-Stieltjes integrals of the form

$$
\int_{0}^{1} \int_{0}^{1} F(x, y) \mathrm{d} C(x, y),
$$

where $C$ is the asymptotic distribution function of the sequence $\left(x_{n}, y_{n}\right)_{n \in \mathbb{N}}$ and is usually referred to as copula (see [7). More precisely, a 2-copula is a function $C:[0,1]^{2} \rightarrow[0,1]$ satisfying the following properties: for every $x, y \in[0,1]$

$$
\begin{gathered}
C(x, 0)=C(0, y)=0, \\
C(x, 1)=x \quad \text { and } \quad C(1, y)=y,
\end{gathered}
$$

and for every $x_{1}, x_{2}, y_{1}, y_{2} \in[0,1]$ with $x_{2} \geq x_{1}$ and $y_{2} \geq y_{1}$

$$
C\left(x_{2}, y_{2}\right)-C\left(x_{2}, y_{1}\right)-C\left(x_{1}, y_{2}\right)+C\left(x_{1}, y_{1}\right) \geq 0 \text {. }
$$

An important property of copulas, which makes the expression in (2) meaningful and which can be derived from the above properties, is that every copula $C$ induces a doubly stochastic measure $\gamma_{C}$ (later on denoted by $\gamma$ when there is no possibility of confusion) on the measurable space $\left([0,1]^{2}, \mathcal{B}\right)$, via the formula

$$
\gamma([a, b] \times[c, d])=C(b, d)-C(b, c)-C(a, d)+C(a, c) .
$$

Moreover, there is a one-to-one correspondence between copulas and doubly stochastic measures. For every copula $C$, the measure $\gamma$ is doubly stochastic in the sense that for every Borel set $B \subset[0,1], \gamma([0,1] \times B)=\gamma(B \times[0,1])=\lambda(B)$, 


\section{AN EXTREMAL PROBLEM IN UNIFORM DISTRIBUTION THEORY}

where $\lambda$ is the Lebesgue measure on $[0,1]$. Conversely, for every doubly stochastic measure $\mu$, there exists a copula $C$ given by

$$
C(u, v)=\mu([0, u] \times[0, v]) .
$$

From the definition one can notice that a probability measure on $\left([0,1]^{2}, \mathcal{B}\right)$ with uniform marginals is doubly stochastic. We refer to [6, 11, 14 for details. Nowadays copulas are very popular in applied probability. In particular, they are used in financial mathematics for modeling dependency structures among different kinds of risks; see [3, 13, 17, 19.

With a slight abuse of notation we write $\int_{0}^{1} \int_{0}^{1} F(x, y) \mathrm{d} C(x, y)$ for the integral with respect to the measure $\gamma$.

Notice, however, that in the calculation of these integrals one can also take advantage of the probabilistic interpretation of a copula, as the joint distribution function of a random vector with uniform marginals. More precisely, consider a random vector $\left(X_{1}, X_{2}\right)$ and suppose that its marginals $F_{1}$ and $F_{2}$ are continuous. By applying the probability integral transformation to each component, the random vector

$$
\left(U_{1}, U_{2}\right)=\left(F_{1}\left(X_{1}\right), F_{2}\left(X_{2}\right)\right)
$$

has uniform marginals. The copula of $\left(X_{1}, X_{2}\right)$ is then defined as the joint cumulative distribution function (cdf) of $\left(U_{1}, U_{2}\right)$ :

$$
C\left(u_{1}, u_{2}\right)=P\left(U_{1} \leq u_{1}, U_{2} \leq u_{2}\right) .
$$

In this setting, the notion of optimal coupling can be stated. Let $\mu, \nu$ be two probability measures on $(\mathbb{R}, \mathcal{B})$. Coupling $\mu$ and $\nu$ means constructing two random variables $X_{1}, X_{2}$ on $\mathbb{R}$ in such a way that $X_{1} \stackrel{\mathrm{d}}{\sim} \mu$ and $X_{2} \stackrel{\mathrm{d}}{\sim} \nu$, with $\stackrel{\mathrm{d}}{\sim}$ denoting equality in distribution. The couple $\left(X_{1}, X_{2}\right)$ is called a coupling of $(\mu, \nu)$. Now, if we introduce a cost function $c(x, y)$ on $\mathbb{R} \times \mathbb{R}$, then the problem of finding

$$
\inf \mathbb{E}\left(c\left(X_{1}, X_{2}\right)\right)
$$

where the pair $\left(X_{1}, X_{2}\right)$ runs over all possible couplings of $(\mu, \nu)$ is called $c$-optimal coupling or Monge-Kantorovich mass transportation problem (see e.g., [18, 28]). Equivalently, this problem can be stated in terms of measures,

$$
\inf \int c(x, y) \mathrm{d} P(x, y),
$$

where the infimum runs over all joint probability measures $P$ on $\mathbb{R} \times \mathbb{R}$ with marginals $\mu$ and $\nu$. For connections of extremal limits and copulas to the theory of optimal transport we refer to [10].

Throughout this paper we consider the equivalent sup problem

$$
\sup \left\{\mathbb{E}\left(c\left(X_{1}, X_{2}\right)\right) \mid X_{1}, X_{2} \text { couplings of } \mu, \nu \text { with } P_{X_{1}}=\mu \text { and } P_{X_{2}}=\nu\right\} \text {. }
$$


Furthermore, we focus our attention on couplings between uniform distributions. In Section 4 we use this approach for solving a specific instance of maximizing the integral (2).

A useful criterion for checking the optimality of a candidate solution is based on the notion of $c$-convexity. A function $f: X \rightarrow \mathbb{R}$ is called $c$-convex if it has a representation $f(x)=\sup _{y}\{c(x, y)+a(y)\}$, for some function $a$. The associated $c$-subdifferential of $f$ at $x$ is then defined through:

$$
\partial_{c} f(x)=\{y \mid f(z)-f(x) \geq c(z, y)-c(x, y) \forall z \in X\}
$$

and $\partial_{c} f=\left\{(x, y) \in X \times Y \mid y \in \partial_{c} f(x)\right\}$.

One observes that $y \in \partial_{c} f(x) \Leftrightarrow \exists a(=a(y)) \in \mathbb{R}$ such that

$$
\psi_{y, a}(x)=c(x, y)+a(y)=f(x) \quad \text { and } \quad \psi_{y, a}(\xi)=c(\xi, y)+a(y) \leq f(\xi),
$$

for every $\xi \in X$, see $[18$.

The dual problem of (3) is given by:

$$
I(c)=\inf \left\{\int h_{1} \mathrm{~d} \mu+\int h_{2} \mathrm{~d} \nu \mid c \leq h_{1}+h_{2}, h_{1} \in L^{1}(\mu) \text { and } h_{2} \in L^{1}(\nu)\right\},
$$

and its study is the basis of the following theorem.

THEOREM 1 (Th. 4.7 from [18]). Let $c$ be such that $c(x, y) \geq a(x)+b(y)$ for some $a \in L^{1}(\mu), b \in L^{1}(\nu)$ and assume finiteness of $I(c)$. Then a pair $\left(X_{1}, X_{2}\right)$ with $X_{1} \stackrel{\mathrm{d}}{\sim} \mu, X_{2} \stackrel{\mathrm{d}}{\sim} \nu$ is an optimal c-coupling between $\mu$ and $\nu$ if and only if

$$
\left(X_{1}, X_{2}\right) \in \partial_{c} f \text { a.s. }
$$

for some c-convex function $f$, equivalently, $X_{2} \in \partial_{c} f\left(X_{1}\right)$ a.s.

\section{Main results}

As already pointed out in [7], the solution of problem (2) depends on the sign of the partial derivative

$$
D_{2}=\frac{\partial^{2} F(x, y)}{\partial x \partial y} .
$$

Special cases have been already studied in the literature, like those described in Fig.1 and Fig. 2. (see [7]). In particular, the upper and lower bounds for the first case are given precisely by the Fréchet-Hoeffding bounds, while in the second case the authors provide a criterion [7. Theorem 7] to find the corresponding extrema. 


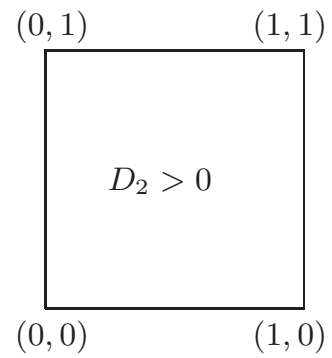

Figure 1.

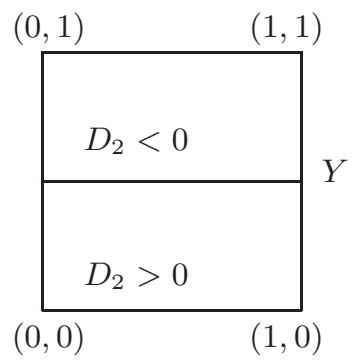

Figure 2.

In this paper we maximize (2) in the special situation described in Fig. 3 as a problem of optimal coupling (see 25) and we provide a criterion for the instance of Fig. 4.

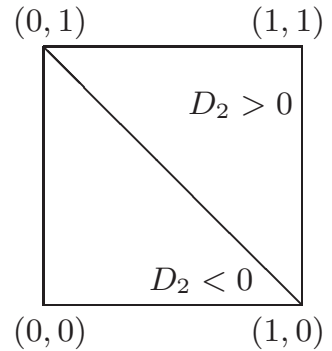

Figure 3.

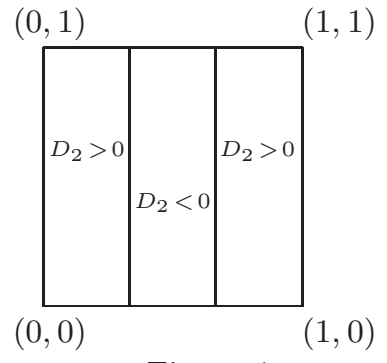

Figure 4.

We start by determining the copula which maximizes (2) when the sign of the second derivative changes as described in Fig. 4. We apply the following criterion [7. Theorem 7].

THEOREM 2. Let us assume that a copula $C(x, y)$ maximizes the integral $\int_{0}^{1} \int_{0}^{1} F(x, y) \mathrm{d} \tilde{C}(x, y)$. Let $\left[X_{1}, X_{2}\right] \times\left[Y_{1}, Y_{2}\right]$ be an interval in $[0,1]^{2}$ such that

$$
C\left(X_{2}, Y_{2}\right)+C\left(X_{1}, Y_{1}\right)-C\left(X_{1}, Y_{2}\right)-C\left(X_{2}, Y_{1}\right)>0
$$

and such that for every interior point $(x, y)$ the mixed second derivative $D_{2}$ has constant sign. Then we have:

(i) if $D_{2}>0$, then

$C(x, y)=\min \left(C\left(x, Y_{2}\right)+C\left(X_{1}, y\right)-C\left(X_{1}, Y_{2}\right), C\left(x, Y_{1}\right)+C\left(X_{2}, y\right)-C\left(X_{2}, Y_{1}\right)\right)$,

(ii) if $D_{2}<0$, then

$C(x, y)=\max \left(C\left(x, Y_{2}\right)+C\left(X_{2}, y\right)-C\left(X_{2}, Y_{2}\right), C\left(x, Y_{1}\right)+C\left(X_{1}, y\right)-C\left(X_{1}, Y_{1}\right)\right)$, for every $(x, y) \in\left[X_{1}, X_{2}\right] \times\left[Y_{1}, Y_{2}\right]$. 
This result can be illustrated by the following pictures, where the symbols $\oplus$ and $\ominus$ in a corner mean that the value of $C$ in that point is taken with positive and negative sign, respectively.

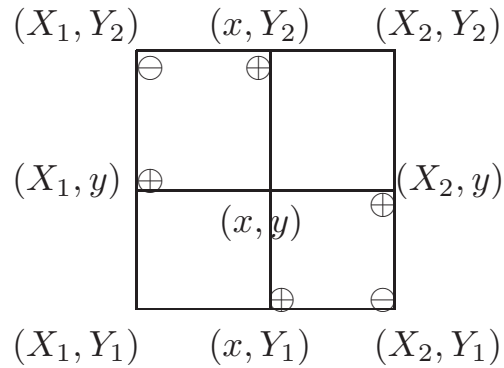

Figure 5 .

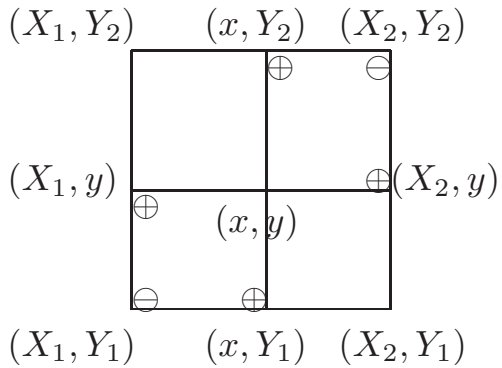

Figure 6.

In order to apply this criterion to the case described in Fig. 4 we divide the unit square $[0,1]^{2}$ into $\left[0, x_{1}\right] \times[0,1],\left[x_{1}, x_{2}\right] \times[0,1]$ and $\left[x_{2}, 1\right] \times[0,1]$, as can be seen in Fig. 7.

Then, following the above statement, if $x \in\left(0, x_{1}\right) \cup\left(x_{2}, 1\right)$, we apply (6) in the same way as in Fig. 5 and if $x \in\left(x_{1}, x_{2}\right)$, we apply (7) as in Fig. 6.

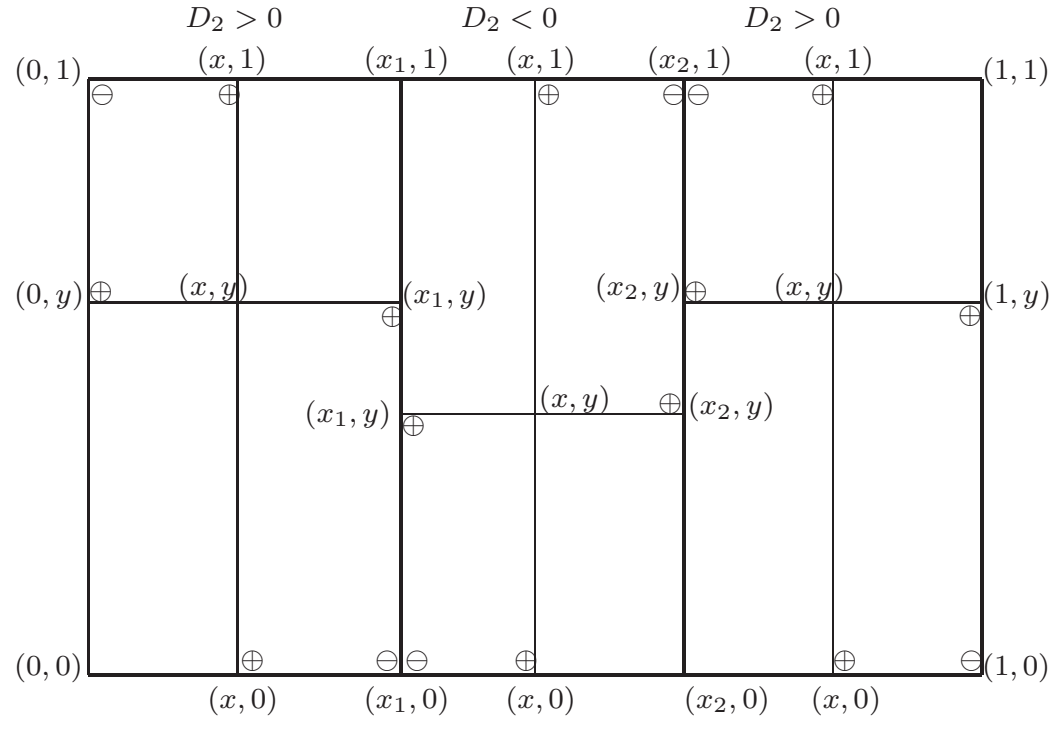

Figure 7 .

Consequently, the following Theorem holds true. 
THEOREM 3. Let $0<x_{1}<x_{2}<1$ and

$$
F(x, y)=\left\{\begin{array}{lll}
F_{1}(x, y) & \text { if } \quad x \in\left(0, x_{1}\right), & \frac{\partial^{2} F_{1}(x, y)}{\partial x \partial y}>0, \\
F_{2}(x, y) & \text { if } \quad x \in\left(x_{1}, x_{2}\right), & \frac{\partial^{2} F_{2}(x, y)}{\partial x \partial y}<0, \\
F_{3}(x, y) & \text { if } \quad x \in\left(x_{2}, 1\right), & \frac{\partial^{2} F_{3}(x, y)}{\partial x \partial y}>0 .
\end{array}\right.
$$

Then the copula maximizing $\int_{0}^{1} \int_{0}^{1} F(x, y) \mathrm{d} \tilde{C}(x, y)$ has the form

$$
C(x, y)= \begin{cases}\min \left(x, h_{1}(y)\right) & \text { if } x \in\left[0, x_{1}\right], \\ \max \left(x+h_{2}(y)-x_{2}, h_{1}(y)\right) & \text { if } x \in\left[x_{1}, x_{2}\right], \\ \min \left(x-x_{2}+h_{2}(y), y\right) & \text { if } x \in\left[x_{2}, 1\right],\end{cases}
$$

where $h_{1}(y)=C\left(x_{1}, y\right)$, and $h_{2}(y)=C\left(x_{2}, y\right)$.

As we will see below, this result implies that in an ideal situation the problem is reduced to the determination of suitable functions $h_{1}$ and $h_{2}$.

Before going on we need to determine $\mathrm{d} C(x, y)$ for the special situation of (9). For this reason let us consider the rectangles

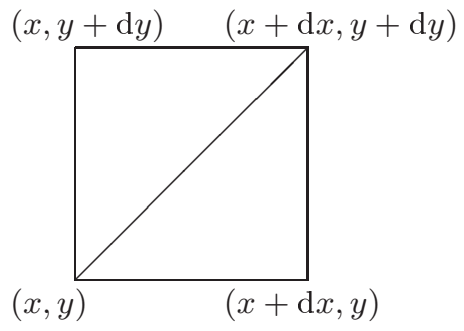

Figure 8.

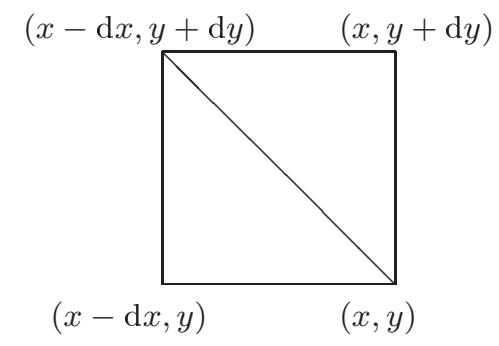

Figure 9.

and the - from the copula induced - measures which are defined by

$$
\gamma_{C}(\mathrm{~d} x, \mathrm{~d} y)=C(x, y)+C(x+\mathrm{d} x, y+\mathrm{d} y)-C(x, y+\mathrm{d} y)-C(x+\mathrm{d} x)
$$

and

$$
\gamma_{C}(\mathrm{~d} x, \mathrm{~d} y)=C(x-\mathrm{d} x, y)+C(x, y+\mathrm{d} y)-C(x-\mathrm{d} x, y+\mathrm{d} y)-C(x, y),
$$

where $(\mathrm{d} x, \mathrm{~d} y)$ stands for the infinitesimal rectangles from Fig. 8 and Fig. 9.

We consider the three regions in Fig. 7 where the second derivative changes sign separately.

(i) $x \in\left(0, x_{1}\right)$.

Then $x=h_{1}(y)$ and $C(x, y)=\min \left(x, h_{1}(y)\right)$. Thus by (10)

$$
\gamma_{C}(\mathrm{~d} x, \mathrm{~d} y)=h_{1}(y)+\left(h_{1}(y)+h_{1}^{\prime}(y) \mathrm{d} y\right)-h_{1}(y)-h_{1}(y)=h_{1}^{\prime}(y) \mathrm{d} y .
$$


V. BALÁŽ - M. R. IACÒ - O. STRAUCH - S. THONHAUSER - R. F. TICHY

(ii) $x \in\left(x_{1}, x_{2}\right)$.

Then $x=x_{2}-h_{2}(y)+h_{1}(y)$ and $C(x, y)=\max \left(x+h_{2}(y)-x_{2}, h_{1}(y)\right)$. Let us observe that

$$
\begin{aligned}
C(x, y+\mathrm{d} y) & =\max \left(x+h_{2}(y+\mathrm{d} y)-x_{2}, h_{1}(y+\mathrm{d} y)\right) \\
& =\max \left(h_{1}(y)+h_{2}^{\prime}(y) \mathrm{d} y, h_{1}(y)+h_{1}^{\prime}(y) \mathrm{d} y\right) \\
& =h_{1}(y)+h_{2}^{\prime}(y) \mathrm{d} y,
\end{aligned}
$$

since for every $(x, y)$ such that $x+h_{2}(y)-x_{2}=h_{1}(y)$ we have

$$
\frac{\mathrm{d} x}{\mathrm{~d} y}+h_{2}^{\prime}(y)=h_{1}^{\prime}(y) \quad \text { and } \quad \frac{\mathrm{d} x}{\mathrm{~d} y}<0 .
$$

Similarly,

$$
\begin{aligned}
C(x-\mathrm{d} x, y) & =\max \left(x-\mathrm{d} x+h_{2}(y)-x_{2}, h_{1}(y)\right) \\
& =\max \left(h_{1}(y)-\mathrm{d} x, h_{1}(y)\right)=h_{1}(y),
\end{aligned}
$$

since $\mathrm{d} x>0$. Thus from (11) we have

$$
\begin{aligned}
\gamma_{C}(\mathrm{~d} x, \mathrm{~d} y) & =h_{1}(y)+\left(h_{1}(y)+h_{2}^{\prime}(y) \mathrm{d} y\right)-h_{1}(y)-h_{1}^{\prime}(y) \mathrm{d} y-h_{1}(y) \\
& =\left(h_{2}^{\prime}(y)-h_{1}^{\prime}(y)\right) \mathrm{d} y .
\end{aligned}
$$

(iii) $x \in\left(x_{2}, 1\right)$. Then

$$
x=x_{2}-h_{2}(y)+y \quad \text { and } \quad C(x, y)=\min \left(x-x_{2}+h_{2}(y), y\right) .
$$

Let us observe that

$$
\begin{aligned}
C(x+\mathrm{d} x, y+\mathrm{d} y) & =\min \left(x+\mathrm{d} x-x_{2}+h_{2}(y+\mathrm{d} y), y+\mathrm{d} y\right) \\
& =\min \left(y+\mathrm{d} x+h_{2}^{\prime}(y) \mathrm{d} y, y+\mathrm{d} y\right)=y+\mathrm{d} y,
\end{aligned}
$$

since for every $(x, y)$ such that $x-x_{2}+h_{2}(y)=y$ we have $\mathrm{d} x+h_{2}^{\prime}(y) \mathrm{d} y=\mathrm{d} y$. Moreover,

$$
\begin{aligned}
C(x, y+\mathrm{d} y) & =\min \left(x-x_{2}+h_{2}(y+\mathrm{d} y), y+\mathrm{d} y\right) \\
& =\min \left(y+h_{2}^{\prime}(y) \mathrm{d} y, y+\mathrm{d} y\right)=y+h_{2}^{\prime}(y) \mathrm{d} y,
\end{aligned}
$$

since $h_{2}^{\prime}(y) \leq 1$ and

$$
C(x+\mathrm{d} x, y)=\min \left(x+\mathrm{d} x-x_{2}+h_{2}(y), y\right)=\min (y+\mathrm{d} x, y)=y,
$$

since $\mathrm{d} x>0$. Therefore with (10) we arrive at

$$
\begin{aligned}
\gamma_{C}(\mathrm{~d} x, \mathrm{~d} y) & =C(x, y)+C(x+\mathrm{d} x, y+\mathrm{d} y)-C(x, y+\mathrm{d} y)-C(x+\mathrm{d} x) \\
& =y+y+\mathrm{d} y-\left(y+h_{2}^{\prime}(y) \mathrm{d} y\right)-y=\left(1-h_{2}^{\prime}(y)\right) \mathrm{d} y .
\end{aligned}
$$


Altogether the measure $\gamma_{C}$ of the infinitesimal rectangles and hence $d C(x, y)$ is given by

$$
\gamma_{C}(\mathrm{~d} x, \mathrm{~d} y)=\left\{\begin{array}{lll}
h_{1}^{\prime}(y) \mathrm{d} y & \text { if } x \in\left[0, x_{1}\right], & x=h_{1}(y), \\
\left(h_{2}^{\prime}(y)-h_{1}^{\prime}(y)\right) \mathrm{d} y & \text { if } x \in\left[x_{1}, x_{2}\right], & x=x_{2}-h_{2}(y)+h_{1}(y), \\
\left(1-h_{2}^{\prime}(y)\right) \mathrm{d} y & \text { if } x \in\left[x_{2}, 1\right], & x=x_{2}-h_{2}(y)+y .
\end{array}\right.
$$

Our next step is to identify situations in which $C$ is indeed a copula.

THEOREM 4. The function $C(x, y)$ defined by (9) is a copula if and only if

(i) $h_{1}(y)$ and $h_{2}(y)$ are increasing;

(ii) $h_{1}(0)=0, h_{2}(0)=0$;

(iii) $h_{1}(1)=x_{1}, h_{2}(1)=x_{2}$;

(iv) $0 \leq h_{1}(y) \leq h_{2}(y) \leq y$;

(v) $0 \leq h_{1}^{\prime}(y) \leq h_{2}^{\prime}(y) \leq 1$.

P r o of. The structure of the proof is as follows: we first prove the necessary condition by showing that if $C$ is a copula, then properties (i)-(v) are satisfied. Then we exploit these properties to show that $C$ is a copula.

Let $C(x, y)$ be a copula and $h_{1}(y)=C\left(x_{1}, y\right)$ and $h_{2}(y)=C\left(x_{2}, y\right)$. Properties (i-(iii) are straightforward. In order to prove (iv), let us consider the rectangle $[x, 1] \times[0,1]$. Since $C(x, y)$ is a copula, we have

$$
C(x, 0)+C\left(1, y_{1}\right)-C\left(x, y_{1}\right)-C(1,0)=y_{1}-C\left(x, y_{1}\right) \geq 0,
$$

and thus $y_{1} \geq C\left(x, y_{1}\right)$.

We proceed in a similar way to prove $(v)$. Let us consider the rectangle

$$
[x, 1] \times\left[y_{1}, y_{2}\right] \text {. }
$$

For an arbitrary copula $C(x, y)$ we have

$$
C\left(x, y_{1}\right)+C\left(1, y_{2}\right)-C\left(x, y_{2}\right)-C\left(1, y_{1}\right)=C\left(x, y_{1}\right)+y_{2}-C\left(x, y_{2}\right)-y_{1} \geq 0 \text {. }
$$

Then

$$
y_{2}-y_{1} \geq C\left(x, y_{2}\right)-C\left(x, y_{1}\right)
$$

and thus $C^{\prime}(x, y) \leq 1$ a.e. This implies $h^{\prime}(y) \leq 1$ a.e. for $h(y)=C(x, y)$ (see also [14, Theorem 2.2.7.] ). Furthermore

$$
h_{1}^{\prime}(y) \leq h_{2}^{\prime}(y)
$$

since for every $(x, y)$ such that $x+h_{2}(y)-x_{2}=h_{1}(y)$ with $x \in\left[x_{1}, x_{2}\right]$ we have $\frac{\mathrm{d} x}{\mathrm{~d} y}+h_{2}^{\prime}(y)=h_{1}^{\prime}(y)$ and $\frac{\mathrm{d} x}{\mathrm{~d} y}<0$. 
On the other hand, it follows from $(v)$ and (15) that $\gamma_{C}(\mathrm{~d} x, \mathrm{~d} y)$ is nonnegative for every $(x, y) \in[0,1]^{2}$ and by (9) that $C(x, 0)=C(0, y)=0$. Thus $C(x, y)$ is a distribution function. We need to show that $C(x, 1)=x$ and $C(1, y)=y$ for every $(x, y) \in[0,1]^{2}$. Indeed we have

$$
C(x, 1)= \begin{cases}\min \left(x, h_{1}(1)\right)=\min \left(x, x_{1}\right)=x & \text { if } x \in\left[0, x_{1}\right], \\ \max \left(x+h_{2}(1)-x_{2}, h_{1}(1)\right)=\max \left(x, x_{1}\right)=x & \text { if } x \in\left[x_{1}, x_{2}\right] \\ \min \left(x-x_{2}+h_{2}(1), 1\right)=\min (x, 1)=x & \text { if } x \in\left[x_{2}, 1\right] .\end{cases}
$$

For $x=1$ we need

$$
C(1, y)=\min \left(1-x_{2}+h_{2}(y), y\right)=y .
$$

Since $h_{2}(1)=x_{2}$, then (18) is equivalent

$$
1-y \geq h_{2}(1)-h_{2}(y),
$$

which holds true, since $h_{2}(1)-h_{2}(y)=(1-y) h_{2}^{\prime}\left(y^{*}\right)$ for some $y^{*} \in(y, 1)$ and the derivative satisfies $(v)$.

Theorem 4 implies the following bounds on candidate functions $h_{1}$ and $h_{2}$.

$$
\begin{aligned}
& \overline{h_{1}}(y)=\left\{\begin{array}{ll}
y & \text { if } y \in\left[0, x_{1}\right], \\
x_{1} & \text { if } y \in\left[x_{1}, 1\right],
\end{array} \quad \underline{h_{1}}(y)= \begin{cases}0 & \text { if } y \in\left[0,1-x_{1}\right], \\
y-\left(1-x_{1}\right) & \text { if } y \in\left[1-x_{1}, 1\right],\end{cases} \right. \\
& \overline{h_{2}}(y)=\left\{\begin{array}{ll}
y & \text { if } y \in\left[0, x_{2}\right], \\
x_{2} & \text { if } y \in\left[x_{2}, 1\right],
\end{array} \quad \underline{h_{2}}(y)= \begin{cases}0 & \text { if } y \in\left[0,1-x_{2}\right], \\
y-\left(1-x_{2}\right) & \text { if } y \in\left[1-x_{2}, 1\right],\end{cases} \right.
\end{aligned}
$$

where

$$
\underline{h_{1}}(y) \leq h_{1}(y) \leq \overline{h_{1}}(y), \quad \underline{h_{2}}(y) \leq h_{2}(y) \leq \overline{h_{2}}(y) .
$$

Now, we return to the integral (2).

THEOREM 5. Let us define a function $G$ by

$$
\begin{aligned}
G:= & G\left(y, h_{1}, h_{2}, h_{1}^{\prime}, h_{2}^{\prime}\right)= \\
& =F_{1}\left(h_{1}(y), y\right) h_{1}^{\prime}(y)+F_{2}\left(x_{2}-h_{2}(y)+h_{1}(y), y\right)\left(h_{2}^{\prime}(y)-h_{1}^{\prime}(y)\right) \\
& +F_{3}\left(x_{2}-h_{2}(y)+y, y\right)\left(1-h_{2}^{\prime}(y)\right) .
\end{aligned}
$$

If $h_{1}, h_{2}$ maximize $\int_{0}^{1} G \mathrm{~d} y$ and satisfy Theorem 4, then

$$
\max _{C(x, y)-\text { copula }} \int_{0}^{1} \int_{0}^{1} F(x, y) \mathrm{d} C(x, y)=\int_{0}^{1} G \mathrm{~d} y .
$$

If not, then we only have the following inequality

$$
\max _{C(x, y) \in \mathcal{C}} \int_{0}^{1} \int_{0}^{1} F(x, y) \mathrm{d} C(x, y) \leq \int_{0}^{1} G \mathrm{~d} y .
$$


The class $\mathcal{C}$ is the set of all copulas of the form (9) with $h_{1}$, $h_{2}$ fulfilling the assumptions of Theorem 4.

P r o of. Let $F$ be a function defined on $[0,1]^{2}$ such that $D_{2}=\frac{\partial^{2} F(x, y)}{\partial x \partial y}$ changes its sign as indicated in Fig. 4. Then the two-dimensional Riemann-Stieltjes integral of $F$ with respect to the copula $C$ defined in (9) is given as follows

$$
\begin{aligned}
\int_{0}^{1} \int_{0}^{1} F(x, y) d C(x, y)= & \int_{0}^{1} F_{1}\left(h_{1}(y), y\right) h_{1}^{\prime}(y) \mathrm{d} y \\
& +\int_{0}^{1} F_{2}\left(x_{2}-h_{2}(y)+h_{1}(y), y\right)\left(h_{2}^{\prime}(y)-h_{1}^{\prime}(y)\right) \mathrm{d} y \\
& +\int_{0}^{1} F_{3}\left(x_{2}-h_{2}(y)+y, y\right)\left(1-h_{2}^{\prime}(y)\right) \mathrm{d} y \\
= & \int_{0}^{1} G d y
\end{aligned}
$$

Since under the assumptions of Theorem 4, $C$ is indeed a copula, the representation from Theorem 3. implies optimality. The second statement is obvious, since the class $\mathcal{C}$ is a subset, due to additional restrictions, of candidate functions $h_{1}, h_{2}$.

REMARK 1. Note that to compute extremes of $\int_{0}^{1} G\left(y, h_{1}, h_{2}, h_{1}^{\prime}, h_{2}^{\prime}\right) \mathrm{d} y$ we can apply calculus of variations (cf [29, p. 33]). In particular, if $\left(h_{1}, h_{2}\right)$ are extrema for the integral $\int_{0}^{1} G\left(y, h_{1}, h_{2}, h_{1}^{\prime}, h_{2}^{\prime}\right) \mathrm{d} y$, then $\left(h_{1}, h_{2}\right)$ satisfy the Euler-Lagrange differential equations

$$
\begin{aligned}
& \frac{\partial G}{\partial h_{1}}-\frac{\mathrm{d}}{\mathrm{d} y} \frac{\partial G}{\partial h_{1}^{\prime}}=0 \\
& \frac{\partial G}{\partial h_{2}}-\frac{\mathrm{d}}{\mathrm{d} y} \frac{\partial G}{\partial h_{2}^{\prime}}=0 .
\end{aligned}
$$

The solution $\left(h_{1}, h_{2}\right)$ to (26) maximizes $\int_{0}^{1} G\left(y, h_{1}, h_{2}, h_{1}^{\prime}, h_{2}^{\prime}\right) \mathrm{d} y$ if

$$
\frac{\partial^{2} G}{\partial h_{1}^{\prime} \partial h_{1}^{\prime}} \leq 0, \quad\left|\begin{array}{ll}
\frac{\partial^{2} G}{\partial h_{1}^{\prime} \partial h_{1}^{\prime}} & \frac{\partial^{2} G}{\partial h_{1}^{\prime} \partial h_{2}^{\prime}} \\
\frac{\partial^{2} G}{\partial h_{2}^{\prime} \partial h_{1}^{\prime}} & \frac{\partial^{2} G}{\partial h_{2}^{\prime} \partial h_{2}^{\prime}}
\end{array}\right| \leq 0 .
$$

REMARK 2. From the optimal copula with representation (9) and the properties of $h_{1}$ and $h_{2}$ from Theorem 4. we can derive the solution of the problem in the vocabulary of optimal couplings as well. Notice that for $x \in\left[0, x_{1}\right)$, $(x, y)$ is mapped to $\left(h_{1}(y), y\right)$. According to Theorem $4, h_{1}$ is monotone increasing and admits an inverse $g_{1}$. For $x \in\left[x_{1}, x_{2}\right)$, we have $(x, y)$ is mapped 
to $\left(x_{2}-\left(h_{2}(y)-h_{1}(y)\right), y\right)$, where $x_{2}-\left(h_{2}(y)-h_{1}(y)\right)$ is monotone decreasing in $y$ with inverse function $g_{2}$. Finally, for $x \in\left[x_{2}, 1\right]$, we have $(x, y) \mapsto$ $\left(x_{2}+y-h_{2}(y), y\right)$, with $x_{2}+y-h_{2}(y)$ increasing in $y$ and inverse $g_{3}$. Therefore we can identify the optimal coupling $(U, \Gamma(U))$ for $U$ uniformly distributed on $[0,1]$ and

$$
\Gamma(x)= \begin{cases}g_{1}(x), & x \in\left[0, x_{1}\right), \\ g_{2}(x), & x \in\left[x_{1}, x_{2}\right), \\ g_{3}(x), & x \in\left[x_{2}, 1\right] .\end{cases}
$$

\section{A piecewise linear cost function}

Let

$$
F(x, y)= \begin{cases}F_{1}(x, y)=\frac{x}{x_{1}} y, & x \in\left(0, x_{1}\right), \\ F_{2}(x, y)=\frac{x_{2}-x}{x_{2}-x_{1}} y, & x \in\left(x_{1}, x_{2}\right), \\ F_{3}(x, y)=\frac{x-x_{2}}{1-x_{2}} y, & x \in\left(x_{2}, 1\right),\end{cases}
$$

with $x$-component as shown in Fig. 10.

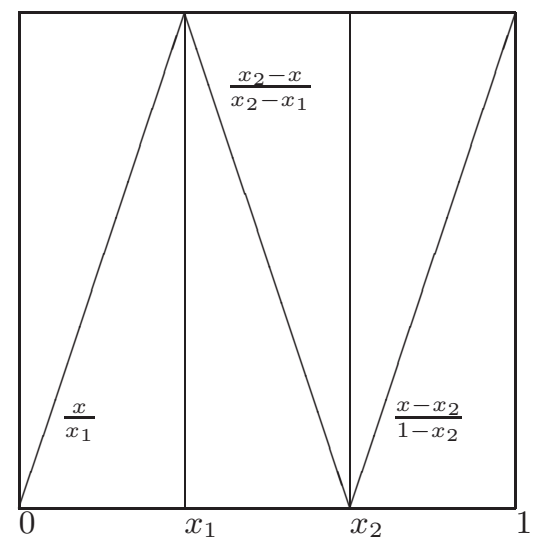

Figure 10.

Towards the construction of $G$ from (21), we identify

$$
\begin{aligned}
& F_{1}\left(h_{1}(y), y\right) h_{1}^{\prime}(y)=\frac{h_{1}}{x_{1}} y h_{1}^{\prime}, \\
& F_{2}\left(x_{2}-h_{2}(y)+h_{1}(y), y\right)\left(h_{2}^{\prime}(y)-h_{1}^{\prime}(y)\right)=\frac{h_{2}-h_{1}}{x_{2}-x_{1}} y\left(h_{2}^{\prime}-h_{1}^{\prime}\right), \\
& F_{3}\left(x_{2}-h_{2}(y)+y, y\right)\left(1-h_{2}^{\prime}(y)\right)=\frac{y-h_{2}}{1-x_{2}} y\left(1-h_{2}^{\prime}\right),
\end{aligned}
$$


such that $G$ takes the form

$$
G=\frac{h_{1}}{x_{1}} y h_{1}^{\prime}+\frac{h_{2}-h_{1}}{x_{2}-x_{1}} y\left(h_{2}^{\prime}-h_{1}^{\prime}\right)+\frac{y-h_{2}}{1-x_{2}} y\left(1-h_{2}^{\prime}\right) .
$$

The associated Euler-Lagrange equations are given by

$$
\begin{aligned}
& \frac{\partial G}{\partial h_{1}}-\frac{\mathrm{d}}{\mathrm{d} y} \frac{\partial G}{\partial h_{1}^{\prime}}=\frac{h_{1}^{\prime}}{x_{1}} y-\frac{h_{2}^{\prime}-h_{1}^{\prime}}{x_{2}-x_{1}} y-\frac{h_{1}}{x_{1}}+\frac{h_{2}-h_{1}}{x_{2}-x_{1}}=0, \\
& \frac{\partial G}{\partial h_{2}}-\frac{\mathrm{d}}{\mathrm{d} y} \frac{\partial G}{\partial h_{2}^{\prime}}=\frac{h_{2}^{\prime}-h_{1}^{\prime}}{x_{2}-x_{1}} y-\frac{1-h_{2}^{\prime}}{1-x_{2}} y-\frac{h_{2}-h_{1}}{x_{2}-x_{1}}+\frac{y-h_{2}}{1-x_{2}}+\frac{y}{1-x_{2}}=0 .
\end{aligned}
$$

Now, adding (29) and (30) and multiplying the sum by $\frac{1-x_{2}}{y}$ we get

$$
h_{1}^{\prime}\left(\frac{1-x_{2}}{x_{1}}\right)+h_{2}^{\prime}=\frac{h_{1}}{y}\left(\frac{1-x_{2}}{x_{1}}\right)+\frac{h_{2}}{y}-1 .
$$

Multiplication of (29) with $\left(\frac{x_{2}-x_{1}}{y}\right)$ gives

$$
h_{1}^{\prime}\left(\frac{x_{2}-x_{1}}{x_{1}}+1\right)-h_{2}^{\prime}=\frac{h_{1}}{y}\left(\frac{x_{2}-x_{1}}{x_{1}}+1\right)-\frac{h_{2}}{y} .
$$

Summing up (31) and (32) we find

and hence

$$
h_{1}^{\prime}=\frac{h_{1}}{y}-x_{1}
$$

$$
h_{2}^{\prime}=\frac{h_{2}}{y}-x_{2}
$$

The general solution $h(y)$ of the differential equation

$$
h^{\prime}=\frac{h}{y}-x,
$$

has the form

$$
h(y)=c y-x y \log y .
$$

From the boundary conditions $h(1)=x$ and $h(0)=0$, we find

$$
h(y)=x y(1-\log y) \quad \text { and } \quad h^{\prime}(y)=x(-\log y) .
$$

Thus

$$
h_{1}(y)=x_{1} y(1-\log y), \quad h_{2}(y)=x_{2} y(1-\log y),
$$

which unfortunately do not satisfy the condition (v) in Theorem 4. 
Finally, $G$ is given through

$$
G=y^{2} \log y\left(\frac{x_{2}}{1-x_{2}}\right)+y^{2}(\log y)^{2}\left(\frac{x_{2}}{1-x_{2}}\right)+y^{2}
$$

which yields the value

$$
\int_{0}^{1} G \mathrm{~d} y=\frac{x_{2}}{1-x_{2}}\left(-\frac{1}{27}\right)+\frac{1}{3}
$$

On the other hand, if $h_{1}(y)=x_{1} y$ and $h_{2}(y)=x_{2} y$, then $G=y^{2}$ and $\int_{0}^{1} G \mathrm{~d} y=\frac{1}{3}$. Thus (37) does not maximize $\int_{0}^{1} G \mathrm{~d} y$.

Note that for $F(x, y)=f(x) y$, with $f(x)$ uniform distribution preserving map (u.d.p.) we have

$$
\begin{aligned}
\max _{x_{n}, y_{n} \text { are u.d. } N \rightarrow \infty} \lim _{N \rightarrow \infty} \frac{1}{N} \sum_{n=1}^{N} f\left(x_{n}\right) y_{n} & =\max _{C(x, y)-\text { copula }} \int_{0}^{1} \int_{0}^{1} F(x, y) \mathrm{d} C(x, y), \\
\max _{x_{n}-\text { u.d, } \Phi-\text { u.d.p. }} \lim _{N \rightarrow \infty} \frac{1}{N} \sum_{n=1}^{N} f\left(x_{n}\right) \Phi\left(x_{n}\right) & =\max _{\Phi-\text { u.d.p. }} \int_{0}^{1} f(x) \Phi(x) \mathrm{d} x .
\end{aligned}
$$

Then (40) is in general smaller than (39). By [7] Corollary 3] for u.d.p. $f(x)$ we have

$$
\max _{\Phi-\text { u.d.p. }} \int_{0}^{1} \int_{0}^{1} f(x) \Phi(x) \mathrm{d} x=\frac{1}{3} .
$$

Therefore in our situation we have $\int_{0}^{1} \int_{0}^{1} F(x, y) \mathrm{d} C(x, y) \geq \frac{1}{3}$.

REMARK 3. The example points out the deficiencies of the variational formulation in the present context. When maximizing (2) it is essential to preserve the uniform distribution property of the marginals. In the formulation via the function (21), which led to the problem from the calculus of variations, this constraint is not present anymore and the maximization takes place over differentiable $h_{1}, h_{2}$. On the other hand the optimal copula $C$ in (9) with $h_{1}(y)=C\left(x_{1}, y\right)$ and $h_{2}(y)=C\left(x_{2}, y\right)$ does not enforce any smoothness properties, which implies that when solving (21) one does not necessarily get an upper bound for (2). The same reasoning suggests that when maximizing (21) over differentiable functions, which fulfill the conditions stated in Theorem 4, one in general derives a lower bound for (2). 


\section{A different approach using coupling}

In this section we consider the particular example

$$
F(x, y)=\sin (\pi(x+y)),
$$

which is of interest since the associated maximization problem could not be solved before by the direct methodology introduced in [7] and above. For this choice $\frac{\partial^{2} F(x, y)}{\partial x \partial y}$ relates to the behavior illustrated in Figure 3. In [9] an upper bound for

$$
\int_{[0,1]^{2}} \sin (\pi(x+y)) \gamma(\mathrm{d} x, \mathrm{~d} y) .
$$

was found by means of a discrete approximation and the Hungarian Algorithm from combinatorial optimization. We will show that the maximizing copula numerically identified in $[9$ is indeed the one maximizing (42). This statement will be an immediate consequence of the following result. It is a variant of a Theorem from [25] and is stated in [10] without complete proof. Here we complement the proof by its mathematical details based on a constructive procedure, see [25, 20], leading to the application of Theorem 1 .

THEOREM 6 (Th. 3.7 from [10]). Let $\mu, \nu$ be the uniform distribution on [0,1] and the cost function $c(x, y)=\phi(x+y)$ with $\phi:[0,2] \rightarrow \mathbb{R}$. In particular we assume that $\phi \in \mathcal{C}^{2}[0,1]$ and that there is $k \in(0,2)$ such that $\phi^{\prime \prime}(x)<0$ for $x \in[0, k)$ and $\phi^{\prime \prime}(x)>0$ for $x \in(k, 2]$. If $\beta \in(0,1)$ denotes the solution to

$$
\phi(2 \beta)-\phi(\beta)=\beta \phi^{\prime}(\beta) \text {, }
$$

then

$$
\Gamma(x)=\left\{\begin{aligned}
\beta-x, & x \in[0, \beta), \\
x, & x \in[\beta, 1],
\end{aligned}\right.
$$

induces by $(U, \Gamma(U))$ for some standard uniformly distributed $U$ an optimal c-coupling between $P$ and $Q$.

REMARK 4. From this Theorem we immediately get a maximizing couple for (42). Namely, $(U, \Gamma(U))$ with $\Gamma$ as above and $\beta=0.75412$ solving

$$
\sin (2 \pi \beta)-\sin (\pi \beta)=\beta \pi \cos (\pi \beta) .
$$

Proof of Theorem 6. For the application of Theorem 1 we need a candidate $c$-convex function:

with

$$
f(x)=f_{1}(x) I_{[0, \beta)}(x)+f_{2}(x) I_{[\beta, 1]}(x),
$$

$$
f_{1}(x)=x \phi^{\prime}(\beta) \quad \text { and } \quad f_{2}(x)=\frac{1}{2}(\phi(2 x)-\phi(2 \beta))+\beta \phi^{\prime}(\beta) .
$$


For checking $c$-convexity, as indicated in (44), introduce for $\xi \in[0,1]$ :

$$
\psi_{\Gamma(x)}(\xi)= \begin{cases}\psi^{1}(\xi), & x \in[0, \beta), \\ \psi^{2}(\xi), & x \in[\beta, 1],\end{cases}
$$

where

$$
\begin{aligned}
& \psi^{1}(\xi)=\phi(\beta-x+\xi)+x \phi^{\prime}(\beta)-\phi(\beta), \\
& \psi^{2}(\xi)=\phi(x+\xi)-\frac{1}{2} \phi(2 x)-\frac{1}{2} \phi(2 \beta)+\beta \phi^{\prime}(\beta) .
\end{aligned}
$$

The first step of the proof towards the $c$-convexity of $f$ is to show

$$
\psi_{\Gamma(x)}(x)=f(x) \forall x \in[0,1]
$$

which follows from the definition of $f$. The second step consists of showing that

$$
\psi_{\Gamma(x)}(\xi) \leq f(\xi) \forall(x, \xi) \in[0,1] \times[0,1] .
$$

At first we need some details on the location of $\beta$. From the definition of $\beta$ and the mean value theorem we obtain $\exists \tau \in(\beta, 2 \beta)$ with $\phi^{\prime}(\tau)=\phi^{\prime}(\beta)$. Since $\phi$ is concave on $[0, k)$ and convex on $(k, 2]$ we see that $\beta<k<\tau<2 \beta$.

In the following we distinguish four situations.

- For $x \in[0, \beta)$ and $\xi \in[0, \beta)$ the functions are given by

$$
\psi_{\Gamma(x)}(\xi)=\phi(\beta-x+\xi)+x \phi^{\prime}(\beta)-\phi(\beta), \quad f(\xi)=\xi \phi^{\prime}(\beta) .
$$

Therefore we need to check:

$$
f(\xi)-\psi_{\Gamma(x)}(\xi)=(\xi-x) \phi^{\prime}(\beta)-(\phi(\beta+\xi-x)-\phi(\beta))=: F_{1}(x, \xi) .
$$

Obviously $F_{1}(x, \xi)=0$ for $(0, \beta),(0,0)$ and $(x, x)$. In general we have by concavity on $[0, k)$ and the definition of $\beta, \phi(2 \beta)=\phi(\beta)+\beta \phi^{\prime}(\beta)$

$$
\phi(\beta)+(\beta+\xi-x-\beta) \phi^{\prime}(\beta) \geq \phi(\beta+\xi-x),
$$

since $\beta+\xi-x \in[0,2 \beta]$, which proves that (43) is positive.

- For $x \in[0, \beta)$ and $\xi \in[\beta, 1]$ the functions are given by

$$
\begin{gathered}
\psi_{\Gamma(x)}(\xi)=\phi(\beta-x+\xi)+x \phi^{\prime}(\beta)-\phi(\beta), \\
f(\xi)=\frac{1}{2}(\phi(2 \xi)-\phi(2 \beta))+\beta \phi^{\prime}(\beta) .
\end{gathered}
$$




\section{AN EXTREMAL PROBLEM IN UNIFORM DISTRIBUTION THEORY}

We need the following to be positive:

$$
\begin{aligned}
& \frac{1}{2}(\phi(2 \xi)-\phi(2 \beta))+\beta \phi^{\prime}(\beta)-\phi(\beta+\xi-x)-x \phi^{\prime}(\beta)+\phi(\beta) \\
& =\frac{1}{2} \phi(2 \xi)+\frac{1}{2} \phi(2 \beta)-x \phi^{\prime}(\beta)-\phi(\beta+\xi-x) \\
& \geq \phi(\xi+\beta)-x \phi^{\prime}(\beta)-\phi(\beta+\xi-x)=: F_{2}(x, \xi),
\end{aligned}
$$

where the equality above follows from the definition of $\beta$ and the inequality follows from convexity since $k<2 \beta \leq 2 \xi$. Observe

$$
F_{2}(x, \xi)=0 \text { for }(0, \xi) \text { and }(\beta, \beta) .
$$

To prove positivity the strategy is as follows, firstly show

$$
F_{2}(x, \xi=\beta) \geq 0 \quad \text { for all } \quad x \in[0, \beta)
$$

and secondly show that

$$
\frac{\partial}{\partial \xi} F_{2}(x, \xi) \geq 0 \quad \text { for all } \quad(x, \xi) \in[0, \beta) \times(\beta, 1) .
$$

Look at

$$
\frac{\partial}{\partial x} F_{2}(x, \xi=\beta)=-\phi^{\prime}(\beta)+\phi^{\prime}(2 \beta-x)
$$

which is zero in $(0, \beta)$ exactly if $x=\hat{x}=2 \beta-\tau<\beta$. Since

$$
0<\phi^{\prime \prime}(\tau)=-\left.\frac{\partial^{2}}{\partial x^{2}} F_{2}(x, \xi=\beta)\right|_{x=\hat{x}}
$$

we have that

$$
F_{2}(\hat{x}, \xi=\beta)=\phi(2 \beta)-(2 \beta-\tau) \phi^{\prime}(\tau)-\phi(\tau)>0
$$

is a maximum, it is positive by convexity $(k<\tau<2 \beta)$. Thus,

$$
F_{2}(x, \xi=\beta) \geq 0 \text { for } x \in[0, \beta) .
$$

Now we can deal with checking the interior,

$$
\frac{\partial}{\partial \xi} F_{2}(x, \xi)=\phi^{\prime}(\beta+\xi)-\phi^{\prime}(\beta+\xi-x) .
$$

Suppose $\beta+\xi-x \geq k$, then by convexity $\phi^{\prime}(\beta+\xi) \geq \phi^{\prime}(\beta+\xi-x)$. On the other hand if $\beta+\xi-x<k$ we have

$$
\phi^{\prime}(\beta+\xi-x)<\phi^{\prime}(\beta)=\phi^{\prime}(\tau) \leq \phi^{\prime}(2 \beta) \leq \phi^{\prime}(\beta+\xi)
$$

since $2 \beta \leq \beta+\xi$. 
- Consider $x \in[\beta, 1]$ and $\xi \in[0, \beta)$, here

$\psi_{\Gamma(x)}(\xi)=\phi(x+\xi)-\frac{1}{2} \phi(2 x)-\frac{1}{2} \phi(2 \beta)+\beta \phi^{\prime}(\beta), \quad f(\xi)=f_{1}(\xi)=\xi \phi^{\prime}(\beta)$,

and we need

$$
\begin{aligned}
\xi \phi^{\prime}(\beta)-\phi(x+\xi)+\frac{1}{2} & \phi(2 x)+\frac{1}{2} \phi(2 \beta)-\beta \phi^{\prime}(\beta) \\
& \geq \phi(x+\beta)-\phi(x+\xi)-(\beta-\xi) \phi^{\prime}(\beta)=: F_{3}(x, \xi)
\end{aligned}
$$

to be positive, the inequality stems from convexity since $2 x \geq 2 \beta>k$. We proceed as before. $F_{3}(x, \xi)=0$ for $(x, \beta)$ and $(\beta, 0)$. At first fix $x=\beta$, $F_{3}(x=\beta, y)=0$ if $y=0$ and $y=\beta$. In between we study

$$
\frac{\partial}{\partial \xi} F_{3}(x=\beta, \xi)=-\phi^{\prime}(\beta+\xi)+\phi^{\prime}(\beta),
$$

which is zero in $(0, \beta)$ exactly if $\xi=\hat{\xi}=\tau-\beta$. Again due to convexity of $\phi$ on $(k, 2]$ we have a maximum in $\hat{\xi}$ and

$$
F_{3}(x=\beta, \hat{\xi})=\phi(2 \beta)-\phi(\tau)-(2 \beta-\tau) \phi^{\prime}(\tau)>0,
$$

such that we have $F_{3}(x=\beta, \xi) \geq 0$ for $\xi \in[0, \beta)$. On the interior we show that

$$
\frac{\partial}{\partial x} F_{3}(x, \xi)=\phi^{\prime}(\beta+x)-\phi^{\prime}(x+\xi) \geq 0 .
$$

If $x+\xi \geq k$ we have from convexity $\phi^{\prime}(\beta+x) \geq \phi^{\prime}(x+\xi)$. If $x+\xi<k$ we have $\beta \leq x+\xi<k \leq \tau<2 \beta \leq \beta+x$ and therefore

$$
\phi^{\prime}(x+\xi) \leq \phi^{\prime}(\beta)=\phi^{\prime}(\tau) \leq \phi^{\prime}(2 \beta) \leq \phi^{\prime}(\beta+x) .
$$

- Let $x \in[\beta, 1]$ and $\xi \in[\beta, 1]$. Here

$$
\begin{aligned}
\psi_{\Gamma(x)}(\xi) & =\phi(x+\xi)-\frac{1}{2} \phi(2 x)-\frac{1}{2} \phi(2 \beta)+\beta \phi^{\prime}(\beta), \\
f(\xi) & =\frac{1}{2}(\phi(2 \xi)-\phi(2 \beta))+\beta \phi^{\prime}(\beta),
\end{aligned}
$$

such that

$$
f(\xi)-\psi_{\Gamma(x)}(\xi)=\frac{1}{2} \phi(2 x)+\frac{1}{2} \phi(2 \xi)-\phi(x+\xi) \geq 0,
$$

is fulfilled by convexity since $x+\xi, 2 x, 2 \xi \geq 2 \beta>k$.

We can conclude that $\psi_{\Gamma(x)}(\xi) \leq f(\xi) \quad \forall(x, \xi) \in[0,1] \times[0,1]$, which according to Theorem 1 shows that the vector $(U, \Gamma(U))$ for $U \stackrel{d}{\sim} \mathcal{U}([0,1])$ yields an optimal coupling. 


\section{AN EXTREMAL PROBLEM IN UNIFORM DISTRIBUTION THEORY}

Acknowledgements. The authors would like to thank an anonymous referee for comments on the manuscript which helped to clarify and improve its presentation.

M.R. Iacò and R.F. Tichy would like to thank the Slovak colleagues for their hospitality during the visit in Bratislava in November 2014.

\section{REFERENCES}

[1] BALÁŽ, V.-MIŠÍK, L.-STRAUCH, O.-TÓTH, J. T.: Distribution functions of ratio sequences, III, Publ. Math. Debrecen 82 (2013), no. 3-4, 511-529.

[2] Distribution functions of ratio sequences, IV, Period. Math. Hungar. 66 (2013) no. $1,1-22$.

[3] BEIGLBÖCK, M.-HENRY-LABORDÈRE, P.-PENKNER, F.: Model-independent bounds for option prices - a mass transport approach, Finance Stoch. 17 (2013), no. 3, $477-501$.

[4] BOSCH, W.: Functions that preserve the uniform distribution of sequences, Trans. Amer. Math. Soc. 307 (1988), no. 1, 143-152.

[5] DRMOTA, M.-TICHY, R. F.: Sequences, Discrepancies and Applications, Lecture Notes in Mathematics Vol. 1651, Springer-Verlag, Berlin, 1997.

[6] DURAnTE, F.-SEMPI, C.: Principles of Copula Theory. CRC/Chapman \& Hall, London, 2015.

[7] FIALOVÁ, J.-STRAUCH, O.: On two-dimensional sequences composed by onedimensional uniformly distributed sequences, Unif. Distrib. Theory 6 (2011), no. 1, 101125 .

[8] GREKOS, G.-STRAUCH, O.: Distribution functions of ratio sequences. II, Unif. Distrib. Theory 2 (2007), no. 1, 53-77.

[9] HOFER, M.-IACÒ, M. R.: Optimal bounds for integrals with respect to copulas and applications, Journal of Optimization Theory and Applications 161 (2014), no. 3, 9991011.

[10] IACÒ, M. R.-TICHY, R. F.-THONHAUSER, S.: Distribution functions, extremal limits and optimal transport. Preprint, 2015.

[11] JAWORSKI, P.-DURANTE, F.-HÄRDLE, W.-RYCHLIK, T. (EDS.): Copula Theory and its Applications. In Proc. Lecture Notes in Statistics Vol. 198. Springer, Heidelberg, 2010.

[12] KUIPERS, L.-NIEDERREITER, H.: Uniform Distribution of Sequences. In: Pure and Applied Mathematics. Wiley-Interscience [John Wiley \& Sons], New York-London-Sydney, 1974 . 


\section{BALÁŽ - M. R. IACÒ - O. STRAUCH - S. THONHAUSER - R. F. TICHY}

[13] MCNEIL, A. J.-FREY, R.-EMBRECHTS, P.: Quantitative Risk Management. Princeton Series in Finance. (Concepts, techniques and tools) Princeton University Press, Princeton, NJ, 2005.

[14] NELSEN, R. B.: An Introduction to Copulas, second edition. Springer Series in Statistics. Springer, New York, 2006.

[15] PILLICHSHAMMER, F.-STEINERBERGER, S.: Average distance between consecutive points of uniformly distributed sequences, Unif. Distrib. Theory 4 (2009), no. 1, 51-67.

[16] PORUBSKÝ, Š.- ¿̌SALÁT, T.-STRAUCH, O.: Transformations that preserve uniform distribution, Acta Arith. 49 (1988), no. 5, 459-479.

[17] PUCCETTI, G.-ÜSCHENDORF, L. R.: Sharp bounds for sums of dependent risks, J. Appl. Probab. 50 (2013), no. 1, 42-53.

[18] ÜSCHENDORF, L. R.: Monge-Kantorovich transportation problem and optimal couplings, Jahresber. Deutsch. Math.-Verein. 109 (2007), no. 3, 113-137.

[19] Mathematical Risk Analysis. Springer Series in Operations Research and Financial Engineering. (Dependence, Risk Bounds, Optimal Allocations and Portfolios). Springer, Heidelberg, 2013.

[20] ÜSCHENDORF, L. R.-UCKELMANN, L.: Numerical and analytical results for the transportation problem of Monge-Kantorovich, Metrika 51 (2000), no. 3, 245-258 (electronic).

[21] STEINERBERGER, S.: Uniform distribution preserving mappings and variational problems, Unif. Distrib. Theory 4 (2009), no. 1, 117-145.

[22] STRAUCH, O.-PORUBSKÝ, Š.: Distribution of Sequences: a Sampler, Schriftenreihe der Slowakischen Akademie der Wissenschaften Vol. 1, Series of the Slovak Academy of Sciences. Peter Lang, Frankfurt am Main, 2005.

[23] STRAUCH, O.-TÓTH, J. T.: Distribution functions of ratio sequences, Publ. Math. Debrecen 58 (2001), no. 4, 751-778.

[24] TICHY, R. F.-WINKLER, R.: Uniform distribution preserving mappings, Acta Arith. 60 (1991), no. 2. 177-18.

[25] UCKELMANN, L.: Optimal couplings between one-dimensional distributions. In: Distributions with given marginals and moment problems (Prague, 1996), Kluwer Acad. Publ., Dordrecht, 1997, pp. 275-281.

[26] VAN DER CORPUT, J. G.: Verteilungsfunktionen I-II. Proc. Akad. Amsterdam 38 (1935), 813-821, 1058-1066.

[27] Verteilungsfunktionen III-VIII, Proc. Akad. Amsterdam 39 (1936), 10-19, 19-26, 149-153, 339-344, 489-494, 579-590.

[28] VILLANI, C.: Optimal Transport, Grundlehren der Mathematischen Wissenschaften [Fundamental Principles of Mathematical Sciences] Vol. 338. Springer-Verlag, Berlin, 2009.

[29] WeInstock, R.: Calculus of Variations. Dover Publications, Inc., New York, 1974. (With applications to Physics and Engineering, Reprint of the 1952 edition.) 


\section{AN EXTREMAL PROBLEM IN UNIFORM DISTRIBUTION THEORY}

Received February 24, 2015

Accepted March 11, 2015
Vladimír Baláž

Institute of Information

Automation and Mathematics

Faculty of Food Technology

Slovak Technical University

Radlinského 12

SK-812 37 Bratislava

SL OVAKIA

E-mail: vladimir.balaz@stuba.sk

\section{Oto Strauch}

Department of Mathematics

Slovak Academy of Sciences

Štefánikova 49

SK-814 73 Bratislava

SLOVAKIA

E-mail: oto.strauch@mat.savba.sk

Maria Rita Iacò

IRIF (LIAFA), Bât. Sophie Germain

Univ. Paris Diderot, Paris 7

8 Place Aurélie Nemours

F-75205 Paris Cedex 13

FRANCE

E-mail: maria-rita.iaco@liafa.univ-paris-diderot.fr

\section{Stefan Thonhauser}

Institute for Analysis and

Computational Number Theory

Graz University of Technology

Kopernikusgasse 24

A-8010 Graz

AUSTRIA

E-mail: stefan.thonhauser@math.tugraz

\section{Robert F. Tichy}

Institute for Analysis and

Computational Number Theory

Graz University of Technology

Steyrergasse 30

A-8010 Graz

AUSTRIA

E-mail: tichy@tugraz.at 